\title{
Análisis de la vulnerabilidad laboral y los determinantes del trabajo decente. El caso de Ecuador 2008-2011*
}

\author{
Recibido: Mayo 25, 2015 - Aceptado: Agosto 25, 2015
}

Doi: dx.doi.org/10.12804/rev.econ.rosario.18.02.2015.01

\author{
Alejandra Villacís ${ }^{\dagger}$ \\ Marcos Reis $\ddagger$
}

\section{Resumen}

El artículo hace un análisis de la vulnerabilidad del mercado laboral medida a través de la iniciativa de trabajo decente de la OIT. Se aplica — para Ecuador en el período 2008-2011 — un modelo derivado de la metodología de pobreza multidimensional que toma en cuenta tanto el bienestar económico como el bienestar social. Como resultados, se encuentra que, para el promedio del período, apenas el $1 \%$ del total de la población ocupada tiene un trabajo decente, mientras que el $67,6 \%$ tiene un trabajo considerado como no decente, en el cual su ingreso laboral no alcanza para cubrir sus necesidades básicas y posee más de cuatro carencias en sus derechos laborales. Los trabajadores vulnerables por mínimos estándares laborales representan el $31 \%$ y los trabajadores vulnerables por salario, el 0,4\%. Mujeres, trabajadores del sector rural y trabajadores informales son los grupos más propensos a no tener un trabajo decente, mientras el trabajador con mayores años de escolaridad, o que trabaja en el sector público, tiene una mayor probabilidad de conseguir un trabajo decente. Para concluir, se discuten recomendaciones de política pública para el aumento del trabajo decente en Ecuador.

* Las opiniones expresadas en este documento son las de los autores y no deben atribuirse a ninguna institución a la que pudieran estar asociados. Los autores agradecen a la Dra. Aracely Ortega Díaz por dirigir la tesis que propició este artículo y a los comentarios y sugerencias del árbitro anónimo de la revista. Los errores son de los autores.

+ MSc. en Economía y Políticas Públicas del Tecnológico de Monterrey. Correo electrónico: aleja_vc@hotmail.com

$\ddagger$ Universidade Federal do Rio de Janeiro (UFRJ).

Para citar este artículo: Villacís, A., \& Reis, M. (julio-diciembre, 2015). Análisis de la vulnerabilidad laboral y los determinantes del trabajo decente. El caso de Ecuador 2008-2011. Revista de Economía del Rosario, 18(2), 157-185. Doi: dx.doi.org/10.12804/rev.econ.rosario.18.02.2015.01 
Palabras clave: estándares laborales: nacionales e internacionales, economía laboral: general, econometría.

Clasificación JEL: J8, C01, J01.

\title{
Analysis of Labor Vulnerability and Determinants of Decent Work. The Case of Ecuador 2008-2011
}

\begin{abstract}
We study labor vulnerability in relation with ILO's approach on decent work. We borrow multidimensional-poverty techniques that encompass economic and social welfare, and use them to compute the prevalence of decent work in Ecuador during 2008-2011. We find that, on average for this period, barely $1 \%$ of the population had a decent job, while $67.6 \%$ had a not-decent job (i.e where perceived wage can not afford basic needs, and where at least four labor rights are missing). Minimum-standards-vulnerable workers account for $31 \%$ of the total, while the wages-vulnerable ones account for $0.4 \%$. Women, rural-sector workers and informal workers, are the groups with the most likelihood of having a not-decent job, while workers with greater years of schooling, and public-sector workers, have the most likelihood of finding a decent job. As a final note, we discuss some public policy recommendations for raising decent-work prevalence rates in Ecuador.
\end{abstract}

Keywords: labor standards: national and international, labor economics: general, econometrics. JEL classification: J8, C01, J01.

\section{Análise da vulnerabilidade laboral e os determinantes do Trabalho Decente. O caso do Equador 2008-2011}

\section{Resumo}

O artigo faz uma análise da vulnerabilidade do mercado laboral medida através da iniciativa de trabalho decente da OIT. Aplica-se, para o Equador no período 2008-2011, um modelo derivado da metodologia de pobreza multidimensional que toma em conta tanto o bem-estar econômico quanto o bem-estar social. Como resultados, se encontra que, para a média do período, apenas o $1 \%$ tem um trabalho considerado como não decente, no qual seu ingresso laboral não alcança para cobrir as suas necessidades básicas e possui mais de quatro carências em seus direitos laborais. Os trabalhadores vulneráveis por mínimas normas laborais representam o $31 \%$ e os trabalhadores vulneráveis por salário, o 0,4\%. Mulheres, trabalhadores do setor rural e trabalhadores informais são os grupos mais propensos a não ter um trabalho decente, enquanto o trabalhador com mais anos de escolaridade, ou que trabalha no setor público, tem uma maior probabilidade de conseguir um trabalho decente. Para concluir, discutem-se recomendações de política pública para o aumento do trabalho decente no Equador.

Palavras-chave: normas laborais: nacionais e internacionais, economia do trabalho: geral, econometria.

Classificação JEL: J8, C01, J01. 


\section{Introducción}

La vulnerabilidad laboral se ha reflejado a lo largo de la historia como el incumplimiento de los derechos de los trabajadores; así mismo, se ha expresado como una manifestación de la precariedad de las condiciones del empleo a las que se enfrentan los individuos en sus respectivos mercados de trabajo. En América Latina, la noción de vulnerabilidad de los trabajadores se ha visto inmersa dentro de los problemas de pobreza y desigualdad que atraviesan cada uno de los países en el continente; el mercado laboral ha estado ligado a vaivenes de los modelos económicos y sociales, y a los diferentes marcos regulatorios de cada gobierno.

En este escenario, la Organización Internacional del Trabajo (OIT) dio a conocer en 1999 la primera iniciativa de trabajo decente, ${ }^{1}$ el cual se consideró como una de las herramientas fundamentales para la erradicación de la pobreza, donde los países deben tomar en cuenta cuatro objetivos estratégicos: creación de empleo, cumplimiento de los derechos de los trabajadores, protección social y fomento del diálogo social.

Con el fin de mejorar las condiciones de los trabajadores, se han desarrollado agendas de política pública que apliquen los principios del trabajo decente dentro de cada país y, para esto, se vio la necesidad de tener un panorama del mercado laboral que revele la situación de vulnerabilidad de la población ocupada. De esta manera, han surgido una serie de propuestas de medición de las condiciones laborales de los trabajadores y creación de índices de trabajo decente adaptados a las regulaciones laborales internacionales en conjunto con el marco legal de cada país.

Sin embargo, no se ha encontrado una metodología que sistematice los múltiples aspectos que conforman las dimensiones del trabajo decente. La elaboración de los índices han planteado problemas en relación con las fórmulas empleadas para ensamblar las variables cualitativas y cuantitativas, y con la ponderación de los indicadores, considerando la igual importancia que debe tener cada norma y derecho social (Ghai, 2003).

El presente estudio contribuye con la literatura llevando a cabo la aplicación de un modelo derivado de la metodología de pobreza multidimensional para medir el trabajo decente bajo los parámetros que establece la OIT a través de un esquema que tome en cuenta tanto la vulnerabilidad por ingresos de

1 Según la OIT (2001), trabajo decente son aspiraciones que tienen los países, en el sentido de que todos se oponen a la discriminación y al trabajo infantil, desean libertad de asociación, participar a través del diálogo social en las decisiones que afectan sus trabajos y vidas, condiciones dignas y seguras de trabajo con remuneraciones adecuadas. 
los trabajadores como el bienestar social en su establecimiento de trabajo. Tal metodología fue primeramente propuesta por Díaz (2013). La autora hizo una aplicación para el caso de México. El presente artículo es el primero en realizar tal metodología para Ecuador.

Este instrumento permite integrar todas las variables que representan a cada una de las dimensiones del concepto, estableciendo una misma ponderación para todas, y clasificará a los trabajadores con base en las carencias que enfrentan dentro de cuatro categorías: personas con un trabajo decente, un trabajo vulnerable por mínimos estándares laborales (MEL), un trabajo no decente y un trabajo vulnerable por salario. Se hace una aplicación empírica con el uso de modelos multilogit para Ecuador en el período 2008-2011.

Se concluye que, para el promedio del período analizado, apenas el 1\% del total de la población ocupada en Ecuador tenía un trabajo decente, mientras que el 67,6\% tenía un trabajo considerado como no decente, en el cual su ingreso laboral no alcanzaba para cubrir sus necesidades básicas y poseían más de cuatro carencias en sus derechos laborales. Los trabajadores vulnerables por MEL representan el $31 \%$ y los trabajadores vulnerables por salario, el $0,4 \%$. Mujeres, trabajadores del sector rural y trabajadores informales son los grupos más propensos a no tener un trabajo decente, mientras el trabajador con mayores años de escolaridad, o que trabaja en el sector público, tiene una mayor probabilidad de conseguir un trabajo decente.

El trabajo está dividido en tres secciones aparte de la presente introducción y la conclusión. El primer apartado discute el marco conceptual, indicadores y estructura de medición del trabajo decente; el segundo presenta una medición de la vulnerabilidad laboral en Ecuador en el período analizado; y, finalmente, el tercer apartado expone los determinantes del trabajo decente a través de un análisis multilogit a fin de identificar cuáles factores son los más importantes para explicar la presencia del trabajo decente.

\section{Marco conceptual, indicadores y estructura de medición del trabajo decente}

La noción de trabajo decente surgió desde mediados de 1940 en la Declaración de Filadelfia, ${ }^{2}$ donde los Estados miembros de la oit se pronunciaban en

2 La Declaración de Filadelfia es una carta incorporada en la Constitución de la OIT que contiene los propósitos y objetivos de la institución, la cual fue promulgada en 1944 y se basa en cuatro principios fundamentales: el trabajo no es mercancía; la libertad de expresión y asociación es esencial; la pobreza en cualquier lugar constituye un peligro para la prospe- 
favor de fomentar programas que promovieran el pleno empleo y leyes que garantizaran a los trabajadores una calidad de vida. La Organización de las Naciones Unidas (ONU) también persiguió estos objetivos y los resaltó en la Declaración Universal de los Derechos Humanos ${ }^{3}$ en 1948. A finales de los noventa, se retoma la discusión, ahora incorporada dentro de un enfoque de desarrollo social y bienestar de las personas para el crecimiento económico sobre escenarios de globalización (OIT, 1999).

Las iniciativas de trabajo decente de la OIT se dieron a conocer por primera vez en la memoria del Director General de la OIT, en la 87ª reunión de la Conferencia Internacional del Trabajo, llevada a cabo en 1999, definiendo a un trabajo decente como un empleo de calidad, que respeta los derechos de los trabajadores y desarrolla progresivamente formas modernas y efectivas de protección social.

\subsection{Dimensiones y aplicación de la politica de trabajo decente}

Las políticas que persiguen el trabajo decente se centran en cuatro elementos esenciales: i) la promoción de los derechos fundamentales en el trabajo; ii) el empleo; iii) la protección social y iv) el diálogo social.

Dentro de la promoción de los derechos fundamentales en el trabajo, se consideran políticas que promuevan la erradicación de la discriminación laboral, del trabajo forzoso y del trabajo infantil. Así mismo, aspectos más específicos, como el número adecuado de horas de trabajo que permitan un equilibrio entre la actividad laboral y la vida familiar, lo que va ligado al derecho de un período de descanso anual, período de maternidad para los padres y demás leyes que establecen las condiciones adecuadas para que los trabajadores estén protegidos en su ámbito laboral.

La promoción del empleo es un objetivo central dentro de las agendas de varios países, que va de la mano con la generación de políticas que impulsen la disminución del desempleo, subempleo, la formalización de los trabajadores y empresas. Bajo este enfoque, se espera que dentro de cada país se amplíen las oportunidades para que las personas puedan encontrar un trabajo productivo y libremente elegido o aceptado. ${ }^{4}$

ridad en todas partes; la guerra contra las carencias se debe desatar con vigor implacable. Ver http://www.ilo.org/ilolex/spanish/iloconst.htm\#annex

3 Artículo 23. Ver http:/ / www.un.org/es/documents/udhr/

4 La Declaración de la OIT relativa a los principios y derechos fundamentales en el trabajo obliga a los Estados miembros a eliminar el trabajo forzoso. Una relación de trabajo 
Al hablar de protección social, se hace referencia al derecho que tienen los trabajadores de resguardar su salud, contar con un sistema de pensiones y un seguro de desempleo. Es decir, establecer los medios de salvaguardia contra la vulnerabilidad y los sucesos imprevistos. Los sistemas de seguridad social permiten proveer de recursos a los trabajadores en caso de desempleo, pérdida de los medios de subsistencia, enfermedad y accidentes laborales, vejez y jubilación e invalidez (OIT, 2012).

Finalmente, el diálogo social se desenvuelve en uno de los tres planos siguientes: entre los empleadores y los trabajadores a propósito de las condiciones de contratación y de trabajo; entre la dirección y los trabajadores de una empresa, sobre el funcionamiento de esta; y entre los interlocutores sociales y las autoridades públicas, sobre la política social y económica (Ghai, 2003).

En cuanto a la aplicación de la política de trabajo decente, su objetivo estratégico es hacer ver este concepto como una política fundamental para que los países alcancen un desarrollo sostenible, tomando en cuenta tanto la cantidad como la calidad del empleo, es decir, la creación de más y mejores puestos de trabajo que estén abiertos para todos por igual y con compensaciones equitativas (Somavia, 2008).

En este sentido, es esencial mostrar a las naciones y a sus gobernantes la importancia de este elemento, enfocándose en la idea de que el empleo es el camino para reducir la pobreza. Así lo afirmó el Director General de la OIT en la 91 a Conferencia Internacional del Trabajo: “La integración explícita del empleo y el trabajo decente en las políticas de crecimiento económico y reducción de la pobreza contribuye a maximizar los beneficios para la población y a asegurar que el crecimiento sea sostenible e inclusivo".

Para dar paso a la ejecución de estas políticas, es necesario determinar cuáles serían los indicadores más eficaces que midan el déficit de trabajo decente que tiene cada país y que logren dar un panorama real del mercado laboral, con el fin de que los gobernantes puedan plantearse objetivos claros acordes con la necesidad y circunstancias propias de la población.

\subsection{Indicadores del trabajo decente y su estructura de medición}

Cuando se analiza el mercado laboral, la mayoría de investigaciones se centran en indicadores que miden el nivel de empleo en los países, tasas de ocupación, desocupación o subempleo. Esos datos no pueden dar un panorama de

debería elegirse libremente y sin que pesen amenazas sobre ella. Ver http:/ /www.ilo.org/ declaration/principles/eliminationofchildlabour/lang--es/index.htm 
la calidad de vida que llevan las personas o hasta qué punto los puestos de trabajo han ayudado a potenciar sus capacidades.

Así mismo, se debe considerar que el trabajo decente es un concepto que integra múltiples aspectos del mercado laboral: a nivel macro relaciona a la economía con la sociedad y a nivel micro, a las empresas y sus trabajadores, con lo cual es necesario establecer una matriz de indicadores que abarque cada una de sus dimensiones.

La OIT construyó un índice compuesto por diversos componentes, como empleo e ingresos, protección social y normas internacionales de trabajo, denominado índice de desarrollo del trabajo decente (IDT) ${ }^{5}$ En este sentido, el IDT sería un equivalente al índice de desarrollo humano (IDH), ${ }^{6}$ ya que ayudaría a ampliar la perspectiva de la economía laboral en dimensiones económicas y sociales (OIT, 2001). Todavía es importante resaltar que dicho índice solo fue un ejercicio específico en el espacio (quince países) y tiempo (1990-2000). De tal modo, puede ser considerado como un esfuerzo significativo de la OIT, pero que cuenta con un tamaño muestral limitado.

El primer intento de medición se realizó en América Latina para el período 1990-2000. Los indicadores utilizados fueron: tasa de desempleo, informalidad, salario industrial y brecha de ingresos entre mujeres y hombres, cobertura de la seguridad social y cantidad de horas trabajadas. De los quince países analizados, se encontraron avances en siete (Chile, Colombia, Costa Rica, El Salvador, Honduras, Panamá y Paraguay); en Perú y Bolivia, las condiciones permanecieron constantes; y en seis países (Argentina, Brasil, Ecuador, México, Uruguay y Venezuela) los indicadores mostraron un retroceso.

Para finales de 2003, se resaltó en las "Conclusiones adoptadas por la Conferencia Internacional del Trabajo en su 91 ${ }^{a}$ reunión" el desafío metodológico que había detrás de la estimación del déficit de trabajo decente, debido a la complejidad al momento de definir y relacionar correctamente las variables que abarquen todas las dimensiones del concepto (OIT, 2003).

5 El índice se trabajó para quince países de América Latina para el período 1990-2000. Se dividió en una parte absoluta que clasificó a los países por las mejoras y bajas de sus indicadores sin considerar la magnitud de los cambios, y en una parte relativa donde se ponderaron los resultados por indicador y luego se sumaron los puntajes, así clasificaron a los países por grupos: los que mejoraron mucho, poco, nada, o empeoraron algo o mucho (OIT, 2008).

$6 \mathrm{El}$ IDH es un índice compuesto que mide el avance promedio conseguido por un país en tres dimensiones básicas del desarrollo humano: disfrutar de una vida larga y saludable, acceso a educación y nivel de vida digno. Lo innovador del IDH fue la creación de una estadística única que serviría como marco de referencia tanto para el desarrollo social como para el económico. Ver http://hdr.undp.org/es/informes/mundial/idh2011/resumen/ 
Autores que profundizaron el tema, como Ghai (2003), intentaron captar todos los componentes de la noción de trabajo decente, como las posibilidades de empleo, un empleo remunerado, las condiciones de trabajo (trabajo nocturno, horas de trabajo, horas de reposo y vacaciones), acceso a seguridad social, trabajo infantil, discriminación en el trabajo, libertad de sindicalización y negociación colectiva. Adicionalmente, Bonnet, Figueiredo y Standig (2003) establecieron una familia de índices de trabajo decente enfocándose en fuentes de información en las que se obtienen datos de distintas facetas de seguridad laboral. Con esto realizaron una base de datos 'mundial de seguridad socioeconómica', la cual se dividió en cinco componentes: tres de nivel macro (agregado), uno de nivel meso (la empresa) y otro de nivel micro (el trabajador individual).

Para 2006, la OIT generó un 'índice global de trabajo decente', construido para 90 países con ocho indicadores relacionados con los cuatro principales componentes del trabajo decente: derechos laborales, oportunidades de empleo, protección y diálogo social. Los resultados en general mostraron una mejoría del déficit de trabajo decente, comparándolo con las variables que se encontraban en el primer índice de trabajo decente establecido en 2001. Sin embargo, se vislumbraron muchas diferencias entre países de altos ingresos en relación con los de bajos ingresos. África subsahariana, Asia Meridional y América Latina presentaron un aumento en términos de calidad de empleo, pero en Asia Oriental el déficit de trabajo decente se redujo más que en las otras regiones (Peek, 2006).

En este punto, es importante recalcar lo afirmado por Ghai (2003 y 2005): no existe como tal un índice de trabajo decente avalado oficialmente por la OIT y recomendado metodológicamente por la Conferencia Internacional de Estadísticos del Trabajo (CIET, cuerpo técnico asesor de la OIT). A tal punto es necesario destacar, como observan Camacho et al. (2012), que en Latinoamérica existe una confusión conceptual generada - donde se asume la existencia de un índice de la OIT - a partir del estudio elaborado por Farné (2003) y promovida por la literatura que de dicho estudio se deriva.

En la actualidad, existen todavía muchas facetas del trabajo decente que no se han podido medir con exactitud debido a la heterogeneidad de los mercados de trabajo, lo que plantea un desafío para diseñar metodologías que complementen las mediciones a nivel micro y capten las características locales. Bajo este contexto, se debe tomar en cuenta la necesidad de que los gobiernos manejen una información clara y sistemática para poder evaluar el tema; así mismo, se tiene que considerar la complementariedad de los indicadores con el marco jurídico de cada país, ya que este aspecto puede generar cambios importantes de los datos a lo largo del tiempo, dado el carácter polifacético del concepto. 


\section{Medición de la vulnerabilidad laboral en Ecuador}

El modelo de trabajo decente en Ecuador es todavía un desafío en materia de políticas públicas y de normativa laboral. En los últimos años, se han impulsado iniciativas por parte del gobierno para mejorar las condiciones del mercado de trabajo, a través de reformas a las leyes laborales con recomendaciones de la OIT con base en la normativa internacional, con el objetivo de mejorar la calidad de vida de la población y resolver los diferentes problemas asociados a las condiciones vulnerables de los trabajadores (Viteri, 2010).

Se han llevado a cabo varios estudios en materia de trabajo decente y diagnósticos del mercado laboral ecuatoriano por parte de organismos como la Oficina Regional de la OIT para América Latina y el Caribe, la Comisión Económica para América Latina y el Caribe (Cepal), entre otros entes no gubernamentales que analizan esta temática. Sin embargo, todavía existe una limitación en materia de indicadores e información estadística que permita dar un panorama real de la situación de los trabajadores ecuatorianos.

Cabe destacar que hasta el presente no existe un consenso en relación con una metodología que mida el déficit de trabajo decente en cada país. Las limitaciones de los índices compuestos se han manifestado en las distintas formas de ponderar los indicadores y en la manera de ensamblar las variables cuantitativas y cualitativas. En este trabajo, se propone hacer uso de una metodología con aplicación general que consolida la medición de las cuatro dimensiones de trabajo decente bajo un modelo teórico que refleje el carácter multidimensional del concepto y permita asignar igual importancia a las variables que representen cada categoría.

La metodología usada será la llevada a cabo por Ortega et al. (2012) y Díaz (2013), la cual será adaptada a las leyes laborales vigentes en el país y se trabajará con información de la Encuesta nacional de empleo, desempleo y subempleo $(E n e m d u)^{7}$ para el período 2008-2011, que son los años disponibles acorde con el nuevo sistema de medición del Instituto Nacional de Estadística y Censos (INEC).

Se realizará una propuesta de índices individuales por dimensión del trabajo decente, para lo cual se utilizarán las variables encontradas en la Enemdu con base en las dimensiones medibles para el mercado laboral ecuatoriano. Así mismo, se llevará a cabo el análisis tomando en cuenta dos componentes: el de indicadores monetarios y el de indicadores de vulnerabilidad por violación a los estándares mínimos de trabajo decente.

7 La Enemdu está diseñada por el INEC para proporcionar información sobre el mercado laboral ecuatoriano. 
Los indicadores monetarios son el salario digno ${ }^{8}$ y el salario básico unificado. ${ }^{9}$ De esta forma, se crearán dos indicadores: el primero, que represente a la población ocupada ${ }^{10}$ que no reciba un ingreso digno y el segundo, que clasifique a las personas que presentan una violación en el derecho del salario básico unificado. El salario promedio de los trabajadores en 2008 fue de USD 324 mensuales, el cual disminuyó en 2009 a USD 316 y aumentó para 2010 a USD 358 y a USD 377 en 2011. La población ocupada que recibía un ingreso menor al salario digno representó el 70,12\% para 2008, cifra que disminuyó al $65,14 \%$ para 2011 , y, dentro de esos porcentajes, los trabajadores que recibían menos del salario básico unificado fueron el $43,87 \%$ para 2008 , cifra que aumentó al 49,18\% en 2009 y bajó al 45,63\% para 2011. Los indicadores de vulnerabilidad seleccionados acorde con la metodología son:

Horas trabajadas: en promedio para el período, el $72 \%$ de la población ocupada mayor a 18 años sufre de un horario excesivo de trabajo según lo que establece la ley, descontando el tiempo legal de horas extras para los cuatro años analizados; y, para los menores de edad, se encontró que un 43,2\% del total también se encuentra en esta categoría.

Seguridad social: para el año 2008, el 71,7\% del total de personas no contaba con afiliación al seguro social ecuatoriano, cifra que fue cambiando con el transcurso de los años debido a las sanciones legales que se impusieron a los empleadores que no cumplieran con esta disposición; así, en 2011 el número de afiliados aumentó y las personas con esta carencia representaron el 58,8\% del total.

Trabajo insuficiente: el total de la población subempleada para el año 2008 representó el $66 \%$, cifra que se incrementó al 69,1\% para 2009 y disminuyó al $65,2 \%$ y al $61,2 \%$ para 2010 y 2011, respectivamente.

Protección a los derechos: el 48 \% de la población ocupada en 2008 a 2011 no cuenta con un contrato por escrito. De la población que no tiene esta carencia, para el año 2011 se encontró que el 40,21\% tiene un contrato permanente o

8 Concepto que nace en Ecuador a partir de 2010, en el que el gobierno busca un salario que permita a las personas vivir dignamente, o sea, lo que una familia necesita para consumir la canasta básica.

9 Es el salario mínimo legal que reciben los trabajadores del sector privado.

10 Ocupadas son aquellas personas de 10 años y más que trabajaron al menos una hora en la semana de referencia o, pese a que no trabajaron, tienen trabajo, del cual estuvieron ausentes por motivos tales como vacaciones, enfermedad, licencia por estudios. Se consideran ocupadas también a aquellas personas que realizan actividades dentro del hogar por un ingreso, aunque las actividades desarrolladas no guarden las formas típicas de trabajo asalariado o independiente (INEC, 2011). 
indefinido; el 29,82\%, un contrato eventual o temporal; el 6,38\%, por horas; y el $23,58 \%$, por jornal.

Respeto a los derechos: para 2008, el 79,6\% de la población ocupada tenía esta carencia; en 2009, aumentó al 81,6 \% y disminuyó al 74,88 \% para 2011; en ese año, el 54,8 \% de trabajadores no contaba con decimotercer sueldo; el 55\% no recibía el decimocuarto sueldo; y el 57,6\% de trabajadores no disponía de vacaciones con goce de sueldo.

Estabilidad en el trabajo: solo el $52 \%$ de la población ocupada cuenta con un contrato por escrito; del total de esta categoría, para el año 2008, el 44,85\% presentaba un trabajo inestable, cifra que subió en 2009 al 49,54 \%, bajó en 2010 al 46,14\% y, para 2011, representó un 44,9\%.

Seguridad en el trabajo: del total de la población ocupada que consideró su trabajo como inseguro, en 2008 representó el 7,76 \%, indicador que disminuyó para 2011 al 5,86 \%; con relación al último año, un 13\% de las personas respondieron que se sentían descontentas por el ambiente perjudicial en su trabajo; el 10,16\% se sentía descontento por tener un trabajo peligroso en la calle; y el $7,92 \%$ consideraba que en su trabajo habían altas posibilidades de accidentes.

Siguiendo la propuesta de Ortega et al. (2012), se hace la agregación de los indicadores formando un indicador de carencias que determinará la violación al cumplimiento de los derechos laborales: horas excesivas, sin seguridad social, trabajo insuficiente, sin contrato de trabajo, no respeto a los derechos, inestabilidad en el trabajo y ambiente perjudicial. Bajo este modelo, se midió la vulnerabilidad laboral en Ecuador utilizando la Enemdu para el período 20082011. Esta encuesta la realiza el INEC de forma mensual, trimestral y anual para la población de 10 años y más.

El INEC utiliza para la Enemdu un esquema de rotación de viviendas, no se trabaja con base en un mismo panel de datos, con lo que las viviendas entrevistadas en los distintos años analizados no son comparables entre sí. Por lo tanto, para el estudio se usaron las encuestas anuales medidas en diciembre de cada año, debido a que estas son las únicas que tienen cobertura a nivel nacional de la población urbano-rural de Ecuador; de esta manera, los resultados pueden dar un panorama de cómo se ha comportado el mercado laboral ecuatoriano en los últimos cuatro años.

Para construir los indicadores de identificación de trabajo decente, se consideró a la población ocupada y se analizaron los resultados por grupos de edad, sexo y dimensiones de vulnerabilidad.

Al medir las dimensiones de vulnerabilidad laboral en Ecuador para la población ocupada de 10 a 65 años de edad en conjunto, se encontraron datos preocupantes. Apenas el 1 \% de la población ocupada tiene un trabajo decente, 
2011 es el año donde la cifra alcanza un mejor resultado con el 1,25\% de los trabajadores.

Por otro lado, la mayoría de la fuerza laboral presenta un trabajo no decente, en 2009 la cifra llegó a un 70,15\% y, para 2011, se dio la cifra más baja con un $63 \%$ de la población ocupada. Es decir, de 5.819 .200 personas que conforman la población ocupada, aproximadamente 3.260 .533 presentaban al menos una violación en sus derechos laborales y no tenían un ingreso suficiente para satisfacer sus necesidades. Los trabajadores vulnerables por MEL forman entre el $30 \mathrm{y}$ $35 \%$ de la población ocupada y los trabajadores vulnerables por salario, menos del 1\%, que, para 2011, representó aproximadamente a 30.613 trabajadores.

De la población con trabajo no decente, se encontró que el 58,86 \% tiene un trabajo no decente extremo para 2011, lo cual representa aproximadamente a 1,8 millones de personas que ganan menos del salario básico unificado y que presentan la violación de cuatro o más de sus derechos laborales. Se denominó al resto de la población con un trabajo no decente moderado, quienes muestran de 1 a 4 carencias y ganan más que el salario básico unificado pero menos del salario digno.

Al examinar los resultados por sexo, se hallaron panoramas similares, sin embargo, en los cuatro años analizados se puede observar que las mujeres presentan un mayor porcentaje de población ocupada con trabajo no decente, entre el 70 y $73 \%$, y los hombres entre un 59 y $66 \%$, caso contrario en la dimensión de vulnerabilidad por MEL, donde los hombres muestran un mayor porcentaje, entre el 30 y $38 \%$, y las mujeres entre un 24 y $29 \%$.

Para el caso del trabajo decente, vemos que las mujeres tienen un mayor porcentaje de trabajadoras bajo esta dimensión para 2008 a 2010, con un 1,25\%, y los hombres entre un 0,80 y $0,95 \%$, pero, para el año 2011, la tendencia cambia y los hombres presentan una mayor población con trabajo decente, con un $1,45 \%$, mientras que las mujeres apenas alcanzan un $0,91 \%$. Y, al igual que la población en general, los ocupados vulnerables por salario son menores del $1 \%$ para ambos sexos.

Y en el caso de trabajo no decente extremo analizado por sexo en los cuatro años calculados, las mujeres manifiestan un mayor porcentaje de población, para 2011 con un $60,41 \%$, mientras que para los hombres el porcentaje de trabajadores con esta condición laboral extrema representó el 54,64 \% para 2011.

En el espacio de los derechos laborales, la población ocupada muestra entre 3 a 5 carencias; para 2011, el 16,5\% de la población presenta al menos una carencia y el 32,2\%, al menos cuatro; analizando las variables por separado para ese año, se encontró que el $72,5 \%$ de la población tiene un horario excesivo de trabajo, el 58,8\% no tiene seguridad social, el 61,2\% está subempleada, el $48,8 \%$ no posee un contrato por escrito, al $74,8 \%$ no se le respetan 
sus derechos laborales legales, el 44,9\% siente inestabilidad en su trabajo y el $5,86 \%$ no siente seguridad en su ambiente laboral.

Pasemos ahora al análisis de las características de la población ocupada por dimensiones. La primera es la edad. La figura 1 nos muestra que la incidencia de trabajo no decente es mucho mayor entre los jóvenes -en especial hasta los 18 años-. En las edades más avanzadas, la incidencia del trabajo no decente, aunque siga muy alta, disminuye. Todavía los clasificados como MEL aumentan mucho. Así, los que se ubican en la categoría de trabajo decente son siempre muy pocos, con un máximo del 2,1\% en el grupo de las personas de 25 a 29 años.

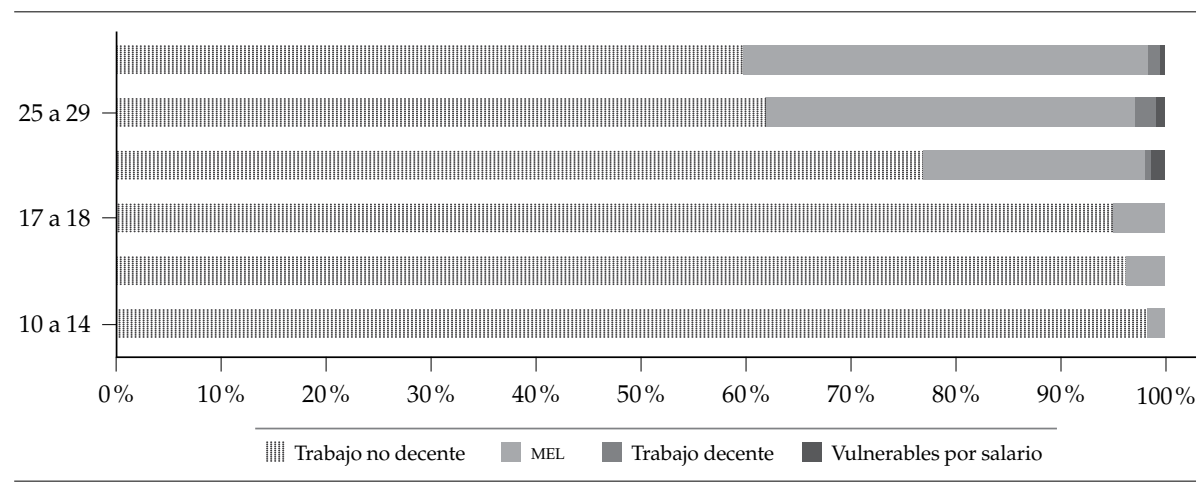

Figura 1. Trabajo decente por grupos de edad (año 2011)

Fuente: cálculos de los autores con datos de la Enemdu.

La figura 2 nos da el panorama de la población ocupada que se encuentra dentro del trabajo no decente y presenta un trabajo no decente extremo. El grupo de 10 a 14 años concentra en su mayoría a niños con un trabajo no decente extremo con el 74,42\%; los trabajadores de 25 a 29 años con un $48,7 \%$; del grupo de 30 a 65 años, que es el que abarca a la mayor parte de la población ocupada, se encontró que 1.326 .068 personas, que representan al $61,11 \%$ del total, tienen un trabajo no decente extremo con 4,7 carencias.

Por otro lado, se llevó a cabo un análisis de la población ocupada según sus sectores de trabajo, con el fin de encontrar las actividades económicas que presentan un mayor grado de vulnerabilidad laboral. Se halló que, del total de población con trabajo no decente, el 38,5\% se ubica en el sector agrícola, seguido del 19,45\%, que se ubica en el área de comercio, y un 10,17\%, que trabaja en industrias manufactureras.

Para el caso de trabajadores vulnerables por MEL, se distribuyen en un mayor número de sectores: el 15,83\% trabaja en el sector comercio; el 15,33\%, en educación; el 10,43\%, en agricultura; y el 9,14\%, en manufactura. Los 


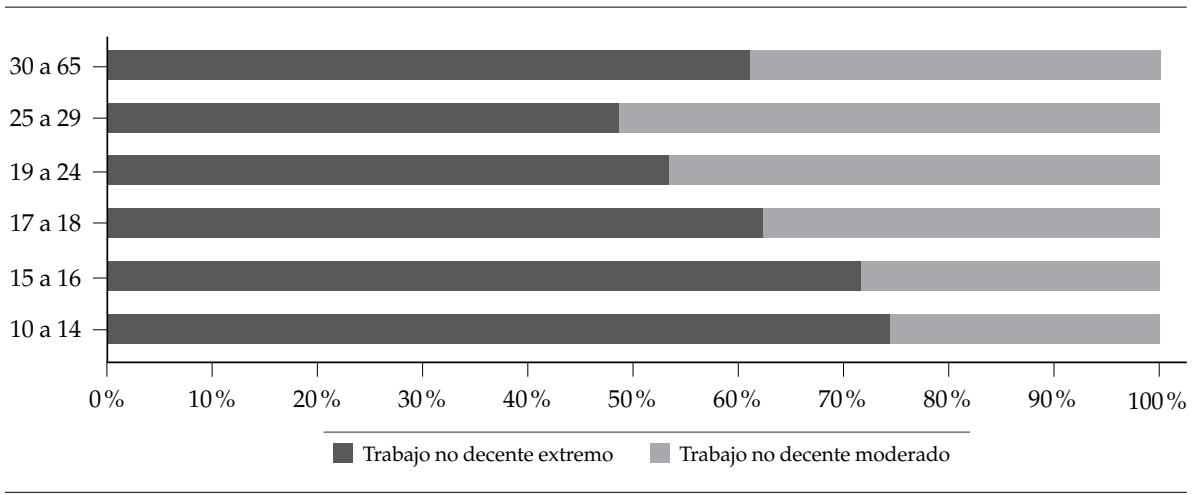

Figura 2. Trabajo no decente moderado y extremo por grupos de edad (año 2011)

Fuente: cálculos de los autores con datos de la Enemdu.

individuos que tienen un trabajo decente se ubican en mayor parte en el área de administración pública, con un 16,45\% del total, seguido de los trabajadores de industrias manufactureras, que representan el 15,58\%. Los trabajadores vulnerables por salario se encuentran principalmente en dos sectores, el manufacturero, con un $24,4 \%$, y en el sector de hoteles y restaurantes, con un $20,6 \%$ del total de ocupados en esa categoría.

De igual forma, para analizar el nivel salarial que percibe la población ocupada según la dimensión en la que se encuentra, se toman en cuenta a los trabajadores de 10 a 65 años en conjunto. Se va a dividir a los trabajadores por sexo para ver las diferencias que existen entre los géneros, ubicándolos según sus ingresos laborales mensuales en cinco categorías salariales, tomando como referencia el salario mínimo, que, para 2011, fue de USD 264 mensuales.

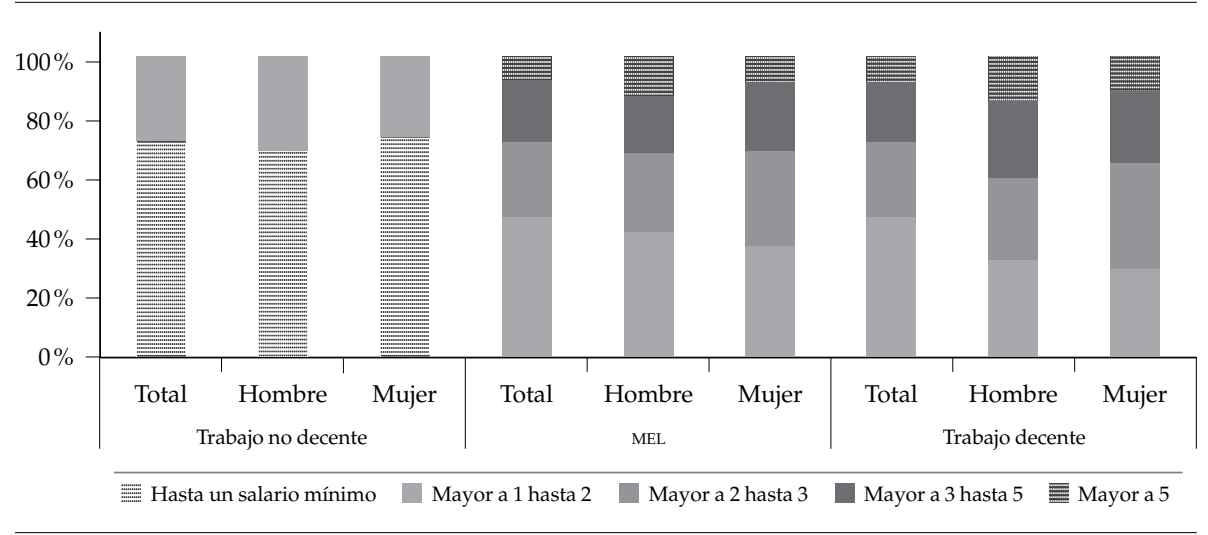

Figura 3. Nivel salarial para la población de 10 a 65 años por sexo Fuente: cálculos de los autores con datos de la Enemdu. 
Siguiendo el mismo esquema, para analizar el grado de escolaridad de la población ocupada por dimensión, se tomó a los trabajadores de 10 a 65 años en conjunto por sexo. En los resultados, se pudo observar que, para las personas con un trabajo no decente, el $46 \%$ tiene educación primaria y el 29,7\% alcanza el nivel secundario, sin hallar mayor diferencia entre género. Para el caso de trabajadores por MEL, se verificó un mayor grado de escolaridad para las mujeres, con un $57,7 \%$ del total que alcanzó el nivel superior universitario, mientras que, para los hombres, fue del $34 \%$ del total que alcanzó dicho nivel. En el segmento de personas con trabajo decente, se encontró el mismo patrón, con un 59,4\% de mujeres que terminaron sus estudios superiores y un $47,4 \%$ del total de hombres para este nivel. Para los trabajadores vulnerables por salario, no existe mayor diferencia entre sexo, el $43,2 \%$ cuenta con educación secundaria y el $23,2 \%$, con educación superior universitaria.

Finalmente, analizando a los trabajadores por su tipo de ocupación, se utilizaron las categorías que se establecen en la Enemdu, distribuyendo a la población de 10 a 65 años por dimensión y por sexo. El 31,8\% de la población ocupada son trabajadores por cuenta propia; el 31,4\%, trabajadores privados; el $11,8 \%$, jornaleros; el 9,6\%, empleados del gobierno; el 9,14\%, trabajadores del hogar no remunerados; ${ }^{11}$ el 3,3\%, patronos; y el 2,4\%, trabajadores domésticos.

\section{Determinantes del trabajo decente}

Luego de analizar los resultados de la vulnerabilidad del mercado laboral ecuatoriano según las dimensiones de trabajo decente, se desea encontrar cuáles son las variables o características de la población ocupada que los ubica dentro de cada categoría, es decir, qué determinantes hacen que una persona tenga o no un trabajo decente.

Existe poca investigación dentro de este tema en específico, los estudios que analizan al trabajo decente se concentran en su mayoría en hallar métodos para medirlo y para construir índices compuestos que clasifiquen a los trabajadores según las variables que conforman el concepto de trabajo decente. Pero estudian a la población como un conjunto homogéneo y no toman en cuenta las diferentes características de los individuos y los factores determinantes que los llevan a obtener un mayor nivel salarial y que garanticen el respeto de sus derechos laborales.

11 Son aquellas personas que trabajan o ayudan en el trabajo, en un negocio o empresa, sin recibir ningún pago por el trabajo realizado. La característica principal de esta categoría está dada por prestar servicios a un miembro del hogar que tiene un negocio familiar (INEC, 2011, p. 85). 


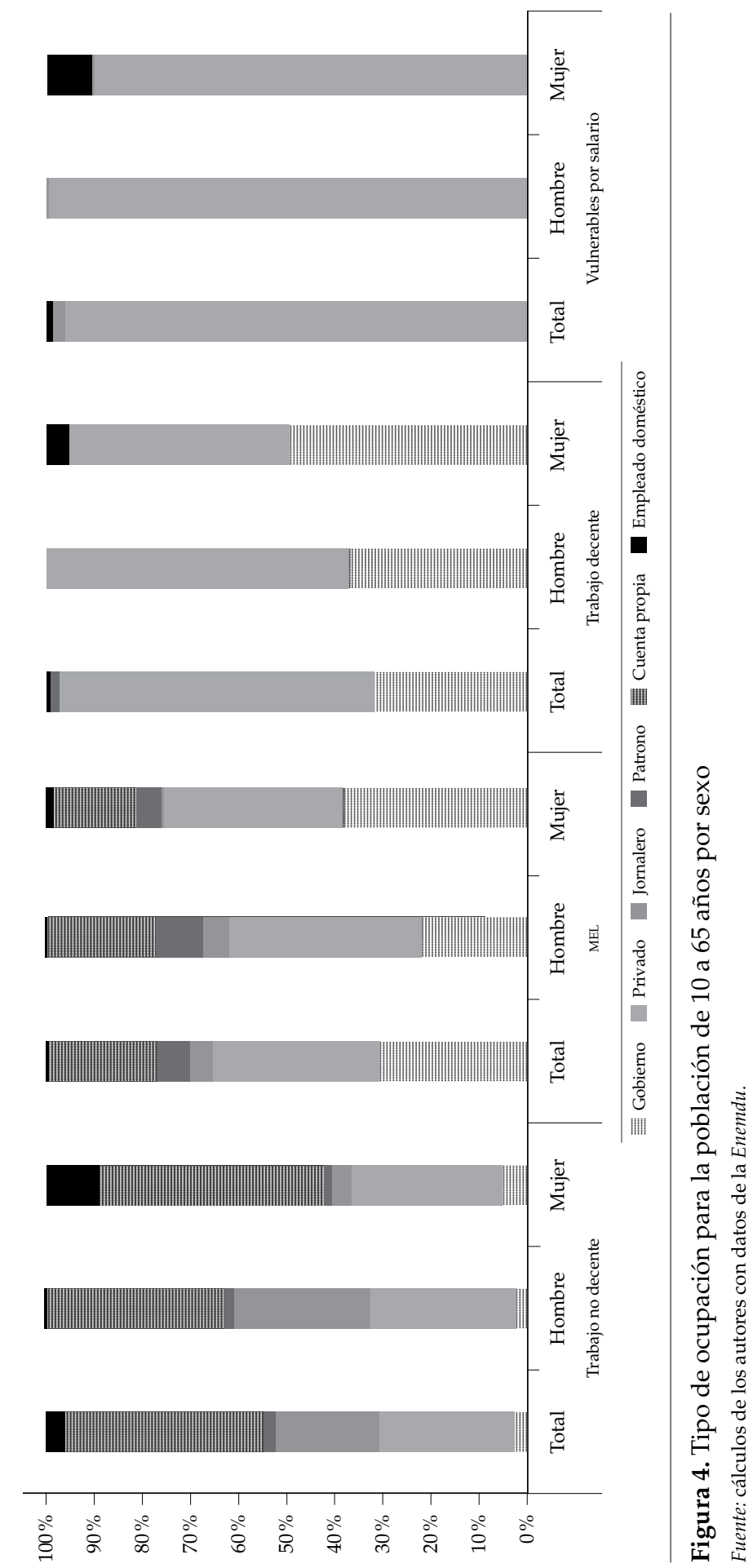


Sin embargo, hay un mayor número de literatura que analiza la vulnerabilidad del mercado laboral por dimensiones separadas: por un lado, autores que investigan los factores determinantes de los diferenciales salariales y violación al salario mínimo, y otros que estudian las variables explicativas que influyen en las condiciones laborales de los trabajadores. ${ }^{12}$

En el presente trabajo, se necesita aplicar una técnica que permita la existencia de un número arbitrario de respuestas, debido a que la población ocupada puede pertenecer a cuatro categorías: tener un trabajo decente, un trabajo vulnerable por MEL, un trabajo no decente o un trabajo que lo haga vulnerable por salario, con lo que la mejor opción es usar un modelo que tome en cuenta múltiples dimensiones.

Theil (1969) desarrolló un modelo multinomial como una extensión del modelo logit, denominado multilogit, el cual permite tener más de dos resultados discretos para la variable dependiente dado un conjunto de variables independientes (las cuales pueden ser categóricas o continuas). Este modelo va a ser aplicado en el presente estudio para estimar los factores que hacen que los trabajadores se ubiquen en determinada dimensión laboral.

\subsection{El modelo econométrico}

El modelo multilogit puede ser pensado como una estimación simultánea de logit binarios, para todas las comparaciones entre las categorías dependientes. El modelo puede ser representado como:

$$
\ln \Omega_{m \mid b}(x)=\ln \frac{\operatorname{Pr}(Y=m \mid x)}{\operatorname{Pr}(Y=b \mid x)}=x \beta_{m \mid b} \text { para } m=1 a J
$$

Donde $b$ es la categoría base, también conocida como grupo comparación. Dado que $\ln \Omega_{b \mid b}(x)=\ln 1=0$, se debe considerar que $\beta_{b \mid b}=0$. Es decir, el resultado de la probabilidad de compararse a sí mismo es siempre 0 , y, por lo tanto, los efectos de las variables independientes también deben ser 0 .

Para el caso del presente estudio, tenemos cuatro categorías de la variable dependiente: si el individuo tiene un trabajo no decente, un trabajo vulnerable por MEL, un trabajo decente o un trabajo vulnerable por salario $(\mathrm{m}=1,2,3$ y 4$)$. Donde se tomará como categoría base a la variable que representa el trabajo

12 En el campo de vulnerabilidad salarial, ver Mincer (1974), Oaxaca (1973), Blinder (1973) y Heckman y Sedlacek (1985). Ya en el campo de la vulnerabilidad de las condiciones y derechos laborales, ver Schmidt y Strauss (1975) y Cassar (2010). 
no decente (1), que es la que tiene el mayor número de observaciones y resultó mejor en las pruebas. Bajo este contexto, el modelo se puede expresar como:

$$
\ln \frac{\operatorname{Pr}\left(Y_{i}=m\right)}{\operatorname{Pr}\left(Y_{i}=1\right)}=\alpha_{m}+\sum_{k=1}^{k} \beta_{m k} X_{i k}
$$

Por lo tanto, para cada caso, habrá $M-1$ predicción de probabilidades, uno para cada categoría con relación a la categoría de referencia. Para realizar la aplicación del modelo al caso de las cuatro dimensiones del trabajo decente, se llevó a cabo un conjunto de pruebas y combinaciones de modelos con las diferentes variables disponibles en la Enemdu y lo que sugiere la literatura. Se tomó en cuenta la significancia de estas dentro de cada categoría, teniendo presente factores de correlación, para escoger el modelo que refleje mejores resultados.

Como variable dependiente vamos a tener las cuatro categorías de trabajo: trabajo no decente (T1), vulnerables por MEL (T2), trabajo decente (T3) y vulnerables por salario (T4). Todas son variables dicotómicas que toman el valor de 1 si el individuo pertenece a determinada categoría.

Las variables independientes se seleccionaron como se mencionó anteriormente, con base en la revisión de literatura y en la disponibilidad de información dentro de la Enemdu; igual que en el estudio de Bhorat, Kanbur y Mayet (2011), se consideró que es necesario establecer tanto un conjunto de características individuales (CI1-CI4) como un conjunto de características que defina al sector o al tipo de empresa (CE1-CE7). ${ }^{13}$

\begin{tabular}{ll}
\hline \multicolumn{1}{c}{ Variable } & \multicolumn{1}{c}{ Definición } \\
\hline (CI1) Escolaridad & Variable continua que mide los años de escolaridad de los trabajadores \\
(CI2) Edad & Edad del trabajador \\
(CI3) Edad2 & Edad del trabajador al cuadrado \\
(CI4) Mujer & Dummy que toma el valor de 1 para mujeres \\
(CE1) Informal & Dummy para el individuo que trabaja en una empresa informal \\
(CE2) Rural & Dummy para el individuo que trabaja en el área rural \\
(CE3) Agricultura & Dummy para el individuo que trabaja en agricultura \\
(CE4) Comercio & Dummy para el individuo que trabaja en comercio \\
\hline
\end{tabular}

13 Para las características del individuo, ver Mincer (1970 y 1974), Oaxaca (1973), Blinder (1973), Heckman y Sedlacek (1985), Neumark (1988), Oaxaca y Ransom (1994), Hirschberg y Slottje (2004). Para las características del sector/empresa, ver Bhorat, Kanbur y Mayet (2011), Oaxaca y Ransom (1994), Hirschberg y Slottje (2004) y Cassar (2010). 
Variable

Definición

(CE5) Manufactura Dummy para el individuo que trabaja en sector de manufactura

(CE6) Público Dummy para el individuo que trabaja en el sector público

(CE7) Privado Dummy para el individuo que trabaja en el sector privado

Una vez establecidas las variables, para la estructuración del modelo, se tomó como categoría base al trabajo no decente, como se mencionó anteriormente. De esta manera se estimarán las variables que determinan que un individuo tenga un trabajo decente a un trabajo no decente, siguiendo el mismo análisis para cada condición laboral:

Vulnerables por mínimos estándares laborales en relación con trabajo no decente

$$
\begin{aligned}
\ln \left(\frac{p(T=2)_{t}}{p(T=1)_{t}}\right) & \\
& =\beta_{20}+\beta_{21}(C I 1)_{t}+\beta_{22}(C I 2)_{t}+\beta_{23}(C I 3)_{t}+\beta_{24}(C I 4)_{t}+\beta_{25}(C E 1)_{t} \\
& +\beta_{26}(C E 2)_{t}+\beta_{27}(C E 3)_{t}+\beta_{28}(C E 4)_{t}+\beta_{29}(C E 5)_{t}+\beta_{210}(C E 6)_{t} \\
& +\beta_{211}(C E 7)_{t}+\varepsilon_{t}
\end{aligned}
$$

Trabajo decente en relación con trabajo no decente

$$
\begin{aligned}
\ln \left(\frac{p(T=3)_{t}}{p(T=1)_{t}}\right) & \\
& =\beta_{30}+\beta_{31}(C I 1)_{t}+\beta_{32}(C I 2)_{t}+\beta_{33}(C I 3)_{t}+\beta_{34}(C I 4)_{t}+\beta_{35}(C E 1)_{t} \\
& +\beta_{36}(C E 2)_{t}+\beta_{37}(C E 3)_{t}+\beta_{38}(C E 4)_{t}+\beta_{39}(C E 5)_{t}+\beta_{310}(C E 6)_{t} \\
& +\beta_{311}(C E 7)_{t}+\varepsilon_{t}
\end{aligned}
$$

Vulnerables por salario en relación con trabajo no decente

$$
\begin{aligned}
\ln \left(\frac{p(T=4)_{t}}{p(T=1)_{t}}\right) & \\
& =\beta_{40}+\beta_{41}(C I 1)_{t}+\beta_{42}(C I 2)_{t}+\beta_{43}(C I 3)_{t}+\beta_{44}(C I 4)_{t}+\beta_{45}(C E 1)_{t} \\
& +\beta_{46}(C E 2)_{t}+\beta_{47}(C E 3)_{t}+\beta_{48}(C E 4)_{t}+\beta_{49}(C E 5)_{t}+\beta_{410}(C E 6)_{t} \\
& +\beta_{411}(C E 7)_{t}+\varepsilon_{t}
\end{aligned}
$$




\subsection{Descripción de la muestra y resultados}

Para el modelo, se trabajó con la encuesta anual de la Enemdu para el año 2011, tomando como análisis solo a la población ocupada de 10 a 65 años, la cual representó una muestra de 27.376 personas, extendiendo a nivel nacional con el factor de expansión a 5.819.200 trabajadores, de los cuales el $39 \%$ son mujeres y el $61 \%$, hombres.

Los años de escolaridad de la población van desde 0 (ningún nivel de instrucción) hasta 21 años, que representa un nivel de posgrado, siendo el promedio 10,58 años, que corresponde al tercer grado de educación secundaria. La edad se tomó como una variable continua considerando a las personas que se incorporan en el mercado laboral desde los 10 años, teniendo como promedio de edad 38 años.

El sector informal abarcó al 52 \% de la población ocupada a nivel nacional. Por su parte, los trabajadores que se ubican en el sector rural representaron el 31 \% del total de la muestra. Como sectores económicos, se escogió la agricultura, comercio y manufactura, que en conjunto abarcaban al $56 \%$ de trabajadores. Y, para las categorías de tipo de ocupación, se consideró a los trabajadores públicos y privados, quienes representan al $40 \%$ del total de la población ocupada. A continuación, la tabla 1 muestra los resultados del modelo, que tomó la condición de trabajo no decente como categoría base.

Tabla 1. Estimación del modelo multilogit con 'trabajo no decente' como línea base $(\mathrm{T}=1)$

\begin{tabular}{lccc}
\hline \multirow{2}{*}{ Variable } & MEL $(\mathrm{T}=2)$ & Trabajo decente $(\mathrm{T}=3)$ & Vulnerable por salario $(\mathrm{T}=4)$ \\
\cline { 2 - 4 } & $\frac{p(T=2)_{t}}{p(T=1)_{t}}$ & $\frac{p(T=3)_{t}}{p(T=1)_{t}}$ & $\frac{p(T=4)_{t}}{p(T=1)_{t}}$ \\
\hline \multirow{2}{*}{ Escolaridad } & $0,1425^{* * *}$ & $0,1156^{* * *}$ & $0,0592^{* *}$ \\
& $(0,0044)$ & $(0,0187)$ & $(0,0251)$ \\
Mujer & $-0,9747^{* * *}$ & $-1,2703^{* * *}$ & $-0,0103$ \\
& $(0,0382)$ & $(0,1613)$ & $(0,1915)$ \\
Edad & $0,1956^{* * *}$ & $0,2163^{* * *}$ & $-0,0473$ \\
& $(0,0095)$ & $(0,0418)$ & $(0,0512)$ \\
Edad ${ }^{2}$ & $-0,0019^{* * *}$ & $-0,0023^{* * *}$ & 0,0005 \\
& $(0,0001)$ & $(0,0005)$ & $(0,0006)$ \\
Informal $^{*}$ & $-0,4768^{* * *}$ & $-2,2267^{* * *}$ & $-1,6576^{* * *}$ \\
& $(0,0418)$ & $(0,4025)$ & $(0,3591)$ \\
\hline
\end{tabular}




\begin{tabular}{lccc}
\hline \multirow{2}{*}{ Variable } & MEL $(\mathrm{T}=2)$ & Trabajo decente $(\mathrm{T}=3)$ & Vulnerable por salario $(\mathrm{T}=4)$ \\
\cline { 2 - 4 } & $p(T=2)_{t}$ & $\frac{p(T=3)_{t}}{p(T=1)_{t}}$ & $\frac{p(T=4)_{t}}{p(T=1)_{t}}$ \\
\hline \multirow{2}{*}{ Rural } & $-0,3361^{* * *}$ & $-0,4504^{* *}$ & $-0,4386$ \\
Agricultura & $(0,0439)$ & $(0,1926)$ & $(0,2521)$ \\
& $-0,8237^{* * *}$ & $-0,3025$ & 0,0602 \\
Comercio & $(0,0567)$ & $(0,2975)$ & $(0,3323)$ \\
\multirow{2}{*}{ Manufactura } & $-0,2129^{* * *}$ & $-0,1456$ & $-0,0713$ \\
& $(0,0454)$ & $(0,2201)$ & $(0,2436)$ \\
Gobierno & $-0,2565^{* * *}$ & 0,1227 & 0,2787 \\
& $(0,0554)$ & $(0,2075)$ & $(0,2351)$ \\
Privado & $2,0406^{* * *}$ & $3,8753^{* * *}$ & $-12,1605$ \\
cons & $(0,0746)$ & $(0,4181)$ & $(534,85)$ \\
& $0,4066^{* * *}$ & $2,6655^{* * *}$ & $3,0806^{* * *}$ \\
\hline
\end{tabular}

${ }^{* * *} \mathrm{p}<0,001,{ }^{* *} \mathrm{p}<0,01 \mathrm{y}{ }^{*} \mathrm{p}<0,05$; representa el error-estándar.

Fuente: cálculos de los autores con datos de la Enemdu.

Fueron realizadas tres pruebas para la validez del modelo: i) likelihood ratio test, ii) wald test y iii) prueba para combinación de categorías dependientes (combine). Los resultados, expuestos en la secuencia, indican que el modelo es significativo.

i) 'Likelihood ratio test'

\begin{tabular}{lrcc}
\hline \multicolumn{1}{c}{ Variables } & \multicolumn{1}{c}{ Chi2 } & Df & p >Chi2 \\
\hline Escolaridad & 1.405 .538 & 4 & 0,000 \\
Sexo & 728,79 & 3 & 0,000 \\
Edad & 755.262 & 3 & 0,000 \\
Edad $^{2}$ & 512.133 & 3 & 0,000 \\
Informal & 203.613 & 3 & 0,000 \\
Agricultura & 391.121 & 3 & 0,000 \\
Comercio & 17.284 & 3 & 0,001
\end{tabular}




\begin{tabular}{lccc}
\hline \multicolumn{1}{c}{ Variables } & Chi2 & Df & p >Chi2 \\
\hline Manufactura & 27.804 & 3 & 0,000 \\
Gobierno & 948.703 & 3 & 0,000 \\
Privado & 257.076 & 3 & 0,000 \\
\hline
\end{tabular}

Ho: todos los coeficientes asociados con la(s) variable(s) dada(s) son 0.

ii) 'Wald test'

\begin{tabular}{lrcc}
\hline \multicolumn{1}{c}{ Variables } & Chi2 & Df & p >Chi2 \\
\hline Escolaridad & 1.023 .323 & 3 & 0,000 \\
Sexo & 669.291 & 3 & 0,000 \\
Edad & 431,72 & 3 & 0,000 \\
Edad $^{2}$ & 280.265 & 3 & 0,000 \\
Informal & 174.096 & 3 & 0,000 \\
Agricultura & 62.339 & 3 & 0,000 \\
Comercio & 216.53 & 3 & 0,000 \\
Manufactura & 22.011 & 3 & 0,000 \\
Gobierno & 25.257 & 3 & 0,000 \\
Privado & 781.981 & 3 & 0,000 \\
\hline
\end{tabular}

Ho: todos los coeficientes asociados con la(s) variable(s) dada(s) son 0.

iii) Prueba para combinación de categorías dependientes (combine)

\begin{tabular}{lrrc}
\hline & Chi2 & Df & p >Chi2 \\
\hline MEL-T_decente & 101.827 & 11 & 0 \\
MEL-Vul_salario & 121.091 & 11 & 0 \\
MEL-T_nodecente & 5.001 .109 & 11 & 0 \\
T_decent-Vul_salario & 38.675 & 11 & 0 \\
T_decente-T_nodecente & 385.317 & 11 & 0 \\
Vul_salario-T_nodecente & 110.409 & 11 & 0 \\
\hline
\end{tabular}

Ho: todos los coeficientes, excepto el intercepto, están asociados con un par de alternativas que son 0 (i. e., las alternativas pueden ser combinadas).

Para la primera variable analizada, escolaridad, los resultados indican que el tener mayores años de escolaridad aumenta la probabilidad de que la persona tenga un trabajo vulnerable por MEL, un trabajo decente o que sea 
vulnerable por salario a tener un trabajo no decente. Lo mismo pasa con la edad, que presenta un coeficiente positivo, mostrando que, a medida que el trabajador tiene mayor edad, la probabilidad de tener un trabajo que no sea no decente es mayor.

Para el caso de la variable que representa a las mujeres, el coeficiente negativo me dice que el hecho de ser mujer disminuye la probabilidad de tener un trabajo vulnerable por MEL y un trabajo decente a tener un trabajo no decente. Los resultados concuerdan con los autores Oaxaca y Ransom (1994) y Hirschberg y Slottje (2004), que toman el factor género como una variable discriminatoria dentro del mercado laboral. Para el caso de los trabajadores vulnerables por salario, la variable también arroja un coeficiente negativo, sin embargo, no se presenta como significativa dentro del modelo.

La variable dummy que representa al sector informal nos muestra que, para los individuos que tienen una condición de actividad informal, es mayor la probabilidad de tener un trabajo no decente. El mismo resultado se refleja para las personas que trabajan en el área rural, que son aproximadamente 1,8 millones de ecuatorianos, quienes representan al 31,2\% del total de la población ocupada, de los cuales el $64,7 \%$ trabaja en la agricultura. ${ }^{14}$

Por otro lado, las variables que constituyen las principales actividades económicas de la población ecuatoriana solo salieron significativas para la relación de trabajo vulnerable por MEL respecto a trabajo no decente. Si una persona trabaja en el sector agrícola, de comercio o manufactura, es menos probable que tenga un trabajo vulnerable por MEL a un trabajo no decente. Finalmente, para las personas que trabajan tanto en el sector público como en el sector privado, es más probable que tengan un trabajo vulnerable por MEL y un trabajo decente a un trabajo no decente.

Una de las principales desventajas de la estimación multilogit es la dificultad que se da para interpretar en el modelo los valores de los coeficientes. Es por eso que, al igual que en los modelos logit, se deben calcular a los momios (odds ratios) para poder entender el efecto de una variable sobre cada categoría (Long, 1997). La figura 5 muestra la razón de momios de las variables independientes para cada variable dependiente.

En la figura 5, se determina la superficie de momios, las variables independientes son cada una representada en una fila separada, y el eje horizontal indica la magnitud relativa de los coeficientes $\beta$ asociados con cada resultado.

14 La pobreza y la desigualdad en Ecuador se han visto con más presencia dentro del sector rural; factores como la falta de escolaridad y la falta de acceso a servicios básicos de vivienda, salud, han hecho de la población que habita en este sector una de las más vulnerables en el país. 


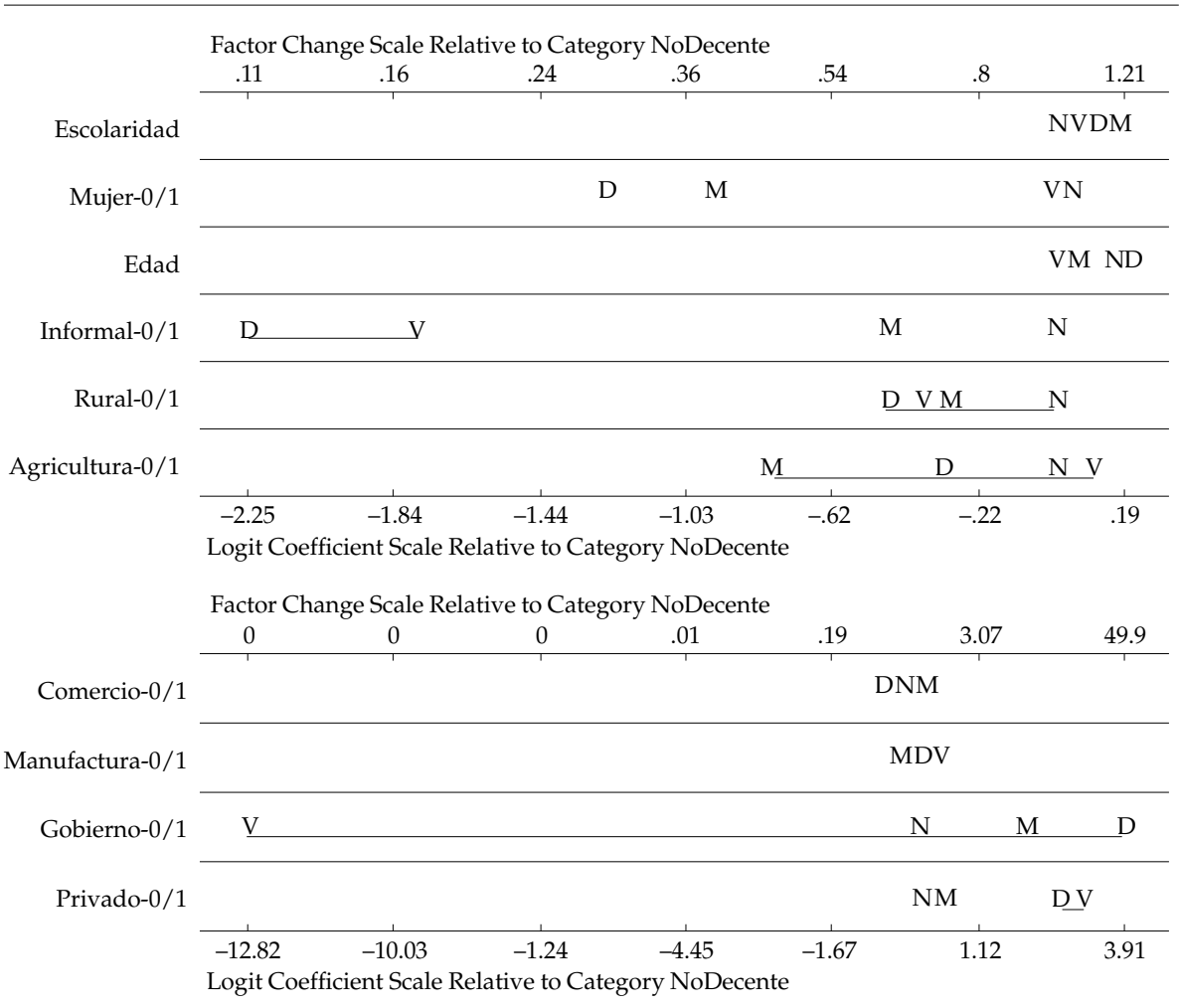

Figura 5. Coeficientes (razón de momios) de las variables independientes para cada variable dependiente

Trabajo decente (D); Trabajo no decente (N); Vulnerables por MEL (M); Vulnerables por salario (V)

Fuente: cálculos de los autores con datos de la Enemdu.

La figura muestra inmediatamente cómo el incremento de una unidad en cada variable afecta a la probabilidad de cada resultado, las letras representan a las categorías de trabajo no decente $(\mathrm{N})$, vulnerables por mínimos estándares laborales (M), trabajo decente (D) y vulnerables por salario (V). Cuando las variables (letras: N, M, D y V) están unidas, se refiere a que el efecto de la variable no es tan grande, como pasa en el caso de la escolaridad, edad, comercio y manufactura. Caso contrario, cuando las variables están separadas, quiere decir que el efecto de estas sobre cada categoría es más grande, como indican las variables que representan a la mujer, sector informal, agricultura y gobierno.

En relación con la dirección de los signos, si una letra está a la derecha de otra, el incremento de la variable independiente hace más probables los efectos sobre la variable dependiente que se encuentra más a la derecha. Para el caso de la variable que representa a la mujer, el hecho de ser mujer hace más 
probable que la persona tenga un trabajo no decente y un trabajo vulnerable por salario a un trabajo decente o vulnerable por MEL.

Si el trabajador es informal, es más probable que tenga un trabajo no decente (N) y un trabajo vulnerable por mínimos estándares laborales (M) a tener un trabajo decente (D) o vulnerable por salario (V). Si la persona trabaja en el sector agrícola, es más probable que tenga un trabajo no decente $(\mathrm{N})$ o un trabajo vulnerable por salario $(\mathrm{V})$. Y si el individuo trabaja en gobierno, es más probable que tenga un trabajo decente (D) a tener cualquier otra categoría (M, N o V).

Finalmente, las líneas que se trazan dentro de cada eje horizontal se dan cuando las variables no son estadísticamente significativas entre las categorías, como es el caso del sector informal para el trabajo decente (D) en relación con el vulnerable por salario (V) y viceversa. Para el caso de la agricultura, como se mostró en los resultados de la figura 5, solo era significativa entre la relación de trabajo vulnerable por MEL y trabajo no decente. Y la variable que representa a los trabajadores del gobierno no es significativa para la relación de trabajadores vulnerables por salario (V) con el resto de categorías (N, M y D).

Para medir la magnitud del efecto de los coeficientes, se pueden obtener los momios para cada relación de categorías. En la tabla 2, se muestran los resultados de la razón de momios por cada variable independiente para la relación entre trabajo vulnerable por MEL, trabajo decente y vulnerables por salario, respecto al trabajo no decente.

Tabla 2. Coeficientes (razón de momios) de las variables independientes para cada variable dependiente

\begin{tabular}{lccc}
\hline \multicolumn{1}{c}{ Variables } & MEL/no decente & Decente/no decente & Vul_sala/no decente \\
\hline Escolaridad & $1,15^{* * * *}$ & $1,12^{* * *}$ & $1,06^{* * *}$ \\
Mujer & $0,38^{* * *}$ & $0,29^{* * *}$ & 0,98 \\
Edad & $1,18^{* * *}$ & $1,21^{* * *}$ & 0,99 \\
Informal & $0,63^{* * *}$ & $0,11^{* * *}$ & $0,17^{* * *}$ \\
Rural & $0,71^{* * *}$ & $0,63^{* * *}$ & 0,68 \\
Agricultura & $0,46^{* * *}$ & 0,73 & 1,10 \\
Comercio & $0,82^{* * *}$ & 0,85 & 0,96 \\
Manufactura & $0,77^{* * *}$ & 1,10 & 1,38 \\
Gobierno & $7,75^{* * *}$ & $49,90^{* * *}$ & 0,00 \\
Privado & $1,50^{* * *}$ & $14,99^{* * *}$ & $22,71^{* * *}$ \\
\hline
\end{tabular}

*** $\mathrm{p}<0,001,{ }^{* *} \mathrm{p}<0,01 \mathrm{y} * \mathrm{p}<0,05$.

Fuente: cálculos de los autores con datos de la Enemdu. 
Por ejemplo, la probabilidad de tener un trabajo vulnerable por MEL en relación con un trabajo no decente es de 1,15 veces más para los trabajadores con mayor años de escolaridad; de la misma manera, la probabilidad de que los individuos tengan un trabajo decente en relación con uno no decente es de 1,12 veces más si tienen una mayor escolaridad y de 1,06 veces para el caso de vulnerables por salario en relación con tener un trabajo no decente, manteniendo el resto de variables constantes. Finalmente, uno de los efectos que resultó con mayor magnitud es el de la variable que representa al trabajo en el sector público. Si una persona trabaja como servidor público, es 49,9 veces más probable que tenga un trabajo decente a un trabajo no decente.

\section{Conclusiones}

Una medición clara del trabajo decente proporcionará a los países nuevas ideas para luchar contra la pobreza laboral y desigualdad, propiciando así la optimización de la agenda en materia de política pública y los objetivos del gobierno. Para lograrlo, los indicadores de trabajo decente deben reflejar tanto las necesidades como las limitaciones de los trabajadores en relación con sus salarios y derechos laborales, de tal manera que se manifiesten todos los factores englobados en este concepto.

La utilización en este trabajo de una metodología análoga a la pobreza multidimensional permite que la medición del trabajo decente abarque todos estos aspectos y le otorgue igual importancia a cada una de sus variables. De esta forma, el modelo nos ayuda a crear un cuerpo conceptual sólido para el análisis del trabajo decente desde esta nueva perspectiva y sirve como herramienta para la evaluación de metas y objetivos de política pública dentro de este campo.

El resultado del análisis para el período 2008-2011 presenta un panorama no favorable para los trabajadores, ya que los resultados de la clasificación por vulnerabilidad laboral muestran que solo un 1,25\% del total de la población ocupada obtiene el salario digno y goza de la totalidad de sus derechos laborales, es decir, cuenta con un trabajo decente. Por otro lado, un $63 \%$ del total de la población ocupada se encuentra en condiciones de trabajo no decente, donde sus salarios no son suficientes para satisfacer sus necesidades básicas. Estos resultados se deben en gran parte a las características que presenta la población ocupada, donde, para el promedio del período, un $66,5 \%$ de trabajadores no cuenta con seguridad social, el 71,9\% sufre de carencia por exceso de horas de trabajo, al 62,9\% no se le respetan sus derechos laborales y el 69,11\% gana menos del salario digno. 
Mujeres, jóvenes (menores de 18 años), trabajadores del sector rural y trabajadores por cuenta propia son identificados como los grupos de riesgo, quienes son más propensos a no tener un trabajo decente. Por otro lado, se observó que el $65 \%$ de personas con un trabajo decente son empleados públicos. De igual manera, la variable que compone al sector público se incorporó en el modelo de estimación y fue la que más efecto tuvo al momento de determinar la razón de momios, mostrando que el hecho de ser un trabajador del gobierno hace 49,9 veces más probable alcanzar un trabajo decente a un trabajo no decente.

Como investigaciones futuras, se propone extender el análisis para un período más largo en Ecuador. El cambio de metodología en la Enemdu limitó el presente análisis para el período 2008-2011. Además, con las políticas favorables que el gobierno ha impulsado, el cual puso entre sus prioridades la mejora de las condiciones laborales de los ciudadanos a través de un salario digno y acceso a seguridad social, es probable que los datos actuales tengan mejores resultados, teniendo en cuenta que los cambios en el sector no son tan inmediatos y se espera que los resultados de las políticas públicas aplicadas en el sentido de mejorar las condiciones laborales se reflejen en el mediano y largo plazo.

Por otro lado, se propone aplicar esta metodología, que sistematiza los múltiples aspectos que conforman las dimensiones del trabajo decente, a otros países, teniendo en cuenta que las variables que miden la vulnerabilidad laboral se pueden adaptar a las condiciones de cada país y a sus marcos regulatorios.

Dados los resultados para cada escenario, y pensando en estudios futuros, es importante analizar a profundidad las características de las personas que tienen un trabajo no decente, haciendo posibles combinaciones entre las variables que incidieron más en esta categoría (mujer - sector informal - área rural), con el fin de establecer la estructura de los trabajadores vulnerables y focalizar aún más los programas de ayuda y su seguimiento.

Finalmente y para la profundización de la dimensión de bienestar económi$\mathrm{co}$, se recomienda estimar el modelo tomando en cuenta diferentes esquemas salariales, como lo son el ingreso promedio de los trabajadores y los salarios mínimos sectoriales. Esto, a su vez, permitirá captar la heterogeneidad que existe entre estados, sectores económicos y diferentes tipos de ocupación.

\section{Referencias}

Bhorat, H., Kanbur, R., \& Mayet, N. (2011). The determinants of minimum wage violations in South Africa. Charles H. Dyson School WP 2011-05. 
Blinder, A. S. (1973). Wage discrimination: reduced form and structural estimates. Journal of Human Resources, 8, 436-455.

Bonnet, F., Figueiredo, J. B., \& Standing, G. (2003). Una familia de índices de trabajo decente. Revista Internacional del Trabajo, 119(1).

Camacho, C., Dussán, L., \& Guataquí, J. (2012), Calidad del trabajo en Bogotá: una aproximación desde el enfoque del trabajo decente. Cuadernos de Desarrollo Económico, 16.

Cassar, L. (2010). Revisiting informality. Evidence from employment characteristics and job satisfaction in Chile. Oxford Poverty \& Human Development Initiative Working Paper, 41.

Díaz, A. O. (enero-junio, 2013). Defining a multidimensional index of decent work for México. The Mexican Journal of Economics and Finance, 8(1).

Farné, S. (2003). Estudio sobre la calidad del empleo en Colombia. Lima: Organización Internacional del Trabajo.

Ghai, D. (2002). Decent work: concepts, models and indicators. International Institute for Labour Studies Discussion Paper. Ginebra: Organización Internacional del Trabajo. DP/139/2002.

Ghai, D. (2003). Decent work: concept and indicators. International Labor Review, 142(2).

Heckman, J. J., \& Sedlacek, G. (1985). Heterogeneity, aggregation, and market wage functions: an empirical model of self-selection in the labor market. The Journal of Political Economy, 93(6), 1077-1125.

Hirschberg, J. G., \& Slottje, D. J. (2004). Bounding estimates of wage discrimination. Research in Labor Economics, 23, 215-233.

Instituto Nacional de Estadísticas y Censos (INEC). (2011). Aspectos metodológicos de la Encuesta nacional de empleo, desempleo y subempleo (Enemdu). Quito, Ecuador.

Long, J. S., \& Freese, J. (1997). Regression models for categorical dependent variables using Stata. Stata Press Publication, College Station, Texas.

Mincer, J. (1970). The distribution of labor incomes: a survey with special reference to the human capital approach. Journal of Economic Literature, 8(1), 1-26.

Mincer, J. (1974). Schooling, experience and earnings. Columbia University Press.

Neumark, D. (1988). Employers' discriminatory behavior and the estimation of wage discrimination. The Journal of Human Resources, 23(3), 279-295.

Oaxaca, R. (1973). Male-female wage differentials in urban labor markets. International Economic Review, 14(3), 693-709.

Oaxaca, R., \& Ransom, M. (1994). On discrimination and the decomposition of wage differentials. Journal of Econometrics, 61, 5-21. 
Organización Internacional del Trabajo (OIT). (1999). Trabajo decente. Memoria del Director General a la $87^{a}$ reunión de la Conferencia Internacional del Trabajo, Ginebra, Suiza.

Organización Internacional del Trabajo (ОIт). (2001). Reducir el déficit de trabajo decente: un desafío global. Memoria del Director General a la 89ª reunión de la Conferencia Internacional del Trabajo, Ginebra, Suiza.

Organización Internacional del Trabajo (ОIT). (2003). Superar la pobreza mediante el trabajo. Conclusiones adoptadas por la Conferencia Internacional del Trabajo en su $91^{\text {a }}$ reunión, Ginebra, Suiza.

Organización Internacional del Trabajo (ОІт). (2008). Medición del trabajo decente. Documento de debate para la reunión tripartita de expertos sobre la medición del trabajo decente, Ginebra, Suiza.

Organización Internacional del Trabajo (ОІт). (2012). Pisos de protección social para la justicia social y una globalización equitativa. Conclusiones adoptadas por la Conferencia Internacional del Trabajo en su 101ª reunión, Ginebra, Suiza. Ortega, A., González, S., Chávez, M., \& Sánchez, A. (2012). Género y desarrollo II. En Propuesta para la medición de la vulnerabilidad laboral según las regulaciones de trabajo decente de la Organización Internacional del Trabajo (ОIT) (pp. 95-115). México: Instituto Nacional de las Mujeres (Inmujeres).

Peek, P. (2006). Decent work deficits around the globe: measuring trends with an index. Mimeo, Geneva.

Schmidt, P., \& Strauss, R. P. (1975). The prediction of occupation using multiple logit models. International Economic Review, 16, 471-486.

Somavia, J. (2008). Declaración de la OIT sobre la justicia social para una globalización equitativa. Conclusiones adoptadas por la Conferencia Internacional del Trabajo en su 101 ${ }^{\text {a }}$ reunión, Ginebra, Suiza.

Theil, H. (1969). A multinomial extension of the linear logit model. International Economic Review, 10(3), 251-259.

Viteri, J. (2010). Trabajo decente: diagnóstico nacional del Ecuador. Programa Laboral de Desarrollo (Plades). Lima, Perú. 
